Geometry $\&$ Topology

Volume 9 (2005) 203-217

Published: 20 January 2005

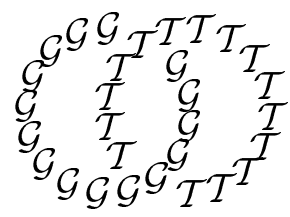

\title{
A stable classification of Lefschetz fibrations
}

\author{
Denis Auroux \\ Department of Mathematics, MIT \\ Cambridge MA 02139, USA \\ Email: auroux@math.mit.edu
}

\begin{abstract}
We study the classification of Lefschetz fibrations up to stabilization by fiber sum operations. We show that for each genus there is a "universal" fibration $f_{g}^{0}$ with the property that, if two Lefschetz fibrations over $S^{2}$ have the same EulerPoincaré characteristic and signature, the same numbers of reducible singular fibers of each type, and admit sections with the same self-intersection, then after repeatedly fiber summing with $f_{g}^{0}$ they become isomorphic. As a consequence, any two compact integral symplectic 4-manifolds with the same values of $\left(c_{1}^{2}, c_{2}, c_{1} \cdot[\omega],[\omega]^{2}\right)$ become symplectomorphic after blowups and symplectic sums with $f_{g}^{0}$.
\end{abstract}

\section{AMS Classification numbers Primary: 57R17}

Secondary: 53D35

Keywords: Symplectic 4-manifolds, Lefschetz fibrations, fiber sums, mapping class group factorizations

Proposed: Tomasz Mrowka

Seconded: Ronald Fintushel, Ronald Stern
Received: 7 December 2004

Accepted: 18 January 2005 


\section{Introduction}

Lefschetz fibrations have been the focus of a lot of attention ever since it was shown by Donaldson that, after blow-ups, every compact symplectic 4-manifold admits such structures [2]. We recall the definition:

Definition 1 A Lefschetz fibration on an oriented compact smooth 4-manifold $M$ is a smooth map $f: M \rightarrow S^{2}$ which is a submersion everywhere except at finitely many non-degenerate critical points $p_{1}, \ldots, p_{r}$, near which $f$ identifies in local orientation-preserving complex coordinates with the model map $\left(z_{1}, z_{2}\right) \mapsto$ $z_{1}^{2}+z_{2}^{2}$.

The smooth fibers of $f$ are compact surfaces, and the singular fibers present nodal singularities; each singular fiber is obtained by collapsing a simple closed loop (the vanishing cycle) in the smooth fiber. The monodromy of the fibration around a singular fiber is given by a positive Dehn twist along the vanishing cycle.

Denoting by $q_{1}, \ldots, q_{r} \in S^{2}$ the images of the critical points (which we will always assume to be distinct), and choosing a reference point $q_{*} \in S^{2} \backslash \operatorname{crit}(f)$, we can characterize the fibration $f$ by its monodromy homomorphism

$$
\psi: \pi_{1}\left(S^{2} \backslash\left\{q_{1}, \ldots, q_{r}\right\}, q_{*}\right) \rightarrow \operatorname{Map}_{g},
$$

where $\operatorname{Map}_{g}=\pi_{0} \operatorname{Diff}^{+}\left(\Sigma_{g}\right)$ is the mapping class group of a genus $g$ surface. It is a classical result (cf. 4]) that the monodromy morphism $\psi$ is uniquely determined up to conjugation by an element of $\mathrm{Map}_{g}$ and the action of a braid on $\pi_{1}\left(S^{2} \backslash\left\{q_{i}\right\}\right)$ by "Hurwitz moves" (see Section 2); moreover, if the fiber genus is at least 2 then the monodromy determines the isomorphism class of the Lefschetz fibration $f$.

The classification of Lefschetz fibrations is a difficult problem (essentially as difficult as the classification of symplectic 4-manifolds), and is only understood in genus 1 and 2 (with some assumptions on the nature of singular fibers in the latter case). It is a classical result of Moishezon and Livne [7] that genus 1 Lefschetz fibrations are all holomorphic, and are classified by the number of irreducible singular fibers (which is a multiple of 12) and the number of reducible singular fibers. More recently, Siebert and Tian [9] have obtained a classification result for genus 2 Lefschetz fibrations without reducible singular fibers and with "transitive monodromy" (a technical assumption which we will not discuss here). Namely, these fibrations are all holomorphic, and are classified by their number of vanishing cycles, which is always a multiple of 10 . In fact, all such 
fibrations can be obtained as fiber sums of two standard holomorphic fibrations $f_{0}$ and $f_{1}$ with respectively 20 and 30 singular fibers.

In higher genus, or even in genus 2 if one allows reducible singular fibers, the classification appears to be much more complicated. However, we can attempt to determine a minimal set of moves (ie, surgery operations) which can be used to relate to each other any two Lefschetz fibrations with the same genus. In this context, we consider stabilization by fiber sums with certain standard fibrations. (The fiber sum of two Lefschetz fibrations is obtained by deleting a neighborhood of a smooth fiber in each of them, and gluing the resulting open manifolds along their boundaries in a fiber-preserving manner). It was shown in [1] that, given two genus 2 symplectic Lefschetz fibrations $f, f^{\prime}$ with the same numbers of singular fibers of each type (irreducible, reducible with genus 1 components, reducible with components of genus 0 and 2), for all large $n$ the fiber sums $f \# n f_{0}$ and $f^{\prime} \# n f_{0}$ are isomorphic. More generally, as a corollary of a recent result of Kharlamov and Kulikov about braid monodromy factorizations [5], a similar result holds for all Lefschetz fibrations with monodromy contained in the hyperelliptic mapping class group.

Our goal is to obtain a similar stabilization result in the general case (without assumptions on the fiber genus or on the monodromy). In this context we must consider pairs of Lefschetz fibrations $f, f^{\prime}$ with the same fiber genus and the same numbers of singular fibers of each type (irreducible, or reducible of type $(h, g-h)$, ie, with components of genera $h$ and $g-h$, for each $\left.0 \leq h \leq \frac{g}{2}\right)$, but we must also place two additional restrictions (which automatically hold when $g \leq 2$ or in the hyperelliptic case). Namely, we must assume that the intersection forms on the total spaces $M$ and $M^{\prime}$ have the same signature, and we must assume that the fibrations $f$ and $f^{\prime}$ admit distinguished sections $s, s^{\prime}$ which represent classes in $H_{2}(M, \mathbb{Z})\left(\operatorname{resp} . H_{2}\left(M^{\prime}, \mathbb{Z}\right)\right)$ with the same selfintersection number $-k$.

Then, we claim that, after repeatedly fiber summing $f$ and $f^{\prime}$ with a certain "universal" Lefschetz fibration $f_{g}^{0}$, constructed in Section 3, we eventually obtain isomorphic Lefschetz fibrations:

Theorem 2 For every $g$ there exists a genus $g$ Lefschetz fibration $f_{g}^{0}$ with the following property. Let $f: M \rightarrow S^{2}$ and $f^{\prime}: M^{\prime} \rightarrow S^{2}$ be two genus $g$ Lefschetz fibrations, each equipped with a distinguished section. Assume that:

(i) the total spaces $M$ and $M^{\prime}$ have the same Euler characteristic and signature;

(ii) the distinguished sections of $f$ and $f^{\prime}$ have the same self-intersection; 
(iii) $f$ and $f^{\prime}$ have the same numbers of reducible fibers of each type.

Then, for all large enough values of $n$, the fiber sums $f \# n f_{g}^{0}$ and $f^{\prime} \# n f_{g}^{0}$ are isomorphic.

A brief remark is in order about assumptions (i) and (ii) in this statement. First, since the Euler characteristic is given by the formula $\chi=4-4 g+r$, where $g$ is the fiber genus and $r$ is the total number of singular fibers, the first part of (i) is equivalent to the requirement that $f$ and $f^{\prime}$ have the same numbers of singular fibers. Moreover, in the hyperelliptic case the assumption on signature can be eliminated, because the signature is given by Endo's formula [3], which involves only the number of singular fibers of each type; however, in general the signature depends on the actual vanishing cycles. It is also worth mentioning that, in general, it is not known whether every Lefschetz fibration admits a section (although there are no known examples without a section). However, all Lefschetz fibrations obtained by blowing up the base points of a pencil (and in particular all those which arise from Donaldson's construction) admit sections of square -1 .

The cases $g=0$ and $g=1$ of Theorem 2 are trivial (in that case no stabilization is needed), and the case $g=2$ is proved in [1] (taking $f_{2}^{0}$ to be the holomorphic genus 2 fibration with 20 singular fibers and total space a rational surface). Thus we will only consider the case $g \geq 3$ in the proof.

As a corollary of Theorem 2 and of Donaldson's result, we have the following statement for integral compact symplectic 4-manifolds (ie, such that $[\omega] \in$ $H^{2}(X, \mathbb{R})$ is the image of an integer cohomology class):

Corollary 3 Let $X, X^{\prime}$ be two integral compact symplectic 4-manifolds with the same $\left(c_{1}^{2}, c_{2}, c_{1} \cdot[\omega],[\omega]^{2}\right)$. Then $X$ and $X^{\prime}$ become symplectomorphic after sufficiently many blowups and symplectic sums with the total space $X_{g}^{0}$ of the fibration $f_{g}^{0}$ (for a suitable genus $g$ ).

This corollary follows from Theorem 2 by considering pencils of the same (large) degree $d$ on $X$ and $X^{\prime}$, and blowing up the $d^{2}[\omega]^{2}$ base points. The resulting Lefschetz fibrations have the same fiber genus (by the assumptions on $c_{1} \cdot[\omega]$ and $\left.[\omega]^{2}\right)$, admit sections of square -1 , and, if $d$ is large enough, can be assumed to contain only irreducible fibers.

The proof of Theorem 2 actually gives a complete classification of Lefschetz fibrations up to fiber sum stabilization. For example, considering only Lefschetz fibrations with irreducible fibers, we have: 
Theorem 4 For every $g \geq 3$ there exist Lefschetz fibrations $f_{g}^{A}, f_{g}^{B}, f_{g}^{C}, f_{g}^{D}$ with the following property: if $f$ is a genus $g$ Lefschetz fibration without reducible singular fibers, and if $f$ admits a section, then there exist integers $a, b, c, d \in \mathbb{Z}$ such that for all large enough values of $n$ the fiber sums $f \# n f_{g}^{0}$ and $(n+a) f_{g}^{A} \#(n+b) f_{g}^{B} \#(n+c) f_{g}^{C} \#(n+d) f_{g}^{D}$ are isomorphic.

The Lefschetz fibrations $f_{g}^{A}, f_{g}^{B}, f_{g}^{C}, f_{g}^{D}$ are constructed in Section 3 (and $f_{g}^{0}$ is in fact nothing but their fiber sum).

The rest of this paper is organized as follows: in Section 2 we review the description of Lefschetz fibrations by mapping class group factorizations; in Section 3 we introduce the concept of universal positive factorization and construct the Lefschetz fibrations $f_{g}^{0}$; and in Sections 4 [5 we prove Theorem 2.

This work was partially supported by NSF grant DMS-0244844.

\section{Mapping class group factorizations}

The monodromy of a Lefschetz fibration can be encoded in a mapping class group factorization by choosing an ordered system of generating loops $\gamma_{1}, \ldots, \gamma_{r}$ for $\pi_{1}\left(S^{2} \backslash\left\{q_{1}, \ldots, q_{r}\right\}\right)$, such that each loop $\gamma_{i}$ encircles only one of the points $q_{i}$ and $\prod \gamma_{i}$ is homotopically trivial. The monodromy of the fibration along each of the loops $\gamma_{i}$ is a Dehn twist $\tau_{i}$; we can then describe the fibration in terms of the relation $\tau_{1} \cdot \ldots \cdot \tau_{r}=1$ in $\operatorname{Map}_{g}$. The choice of the loops $\gamma_{i}$ (and therefore of the twists $\tau_{i}$ ) is of course not unique, but any two choices differ by a sequence of Hurwitz moves exchanging consecutive factors: $\tau_{i} \cdot \tau_{i+1} \rightarrow\left(\tau_{i+1}\right)_{\tau_{i}^{-1}} \cdot \tau_{i}$ or $\tau_{i} \cdot \tau_{i+1} \rightarrow \tau_{i+1} \cdot\left(\tau_{i}\right)_{\tau_{i+1}}$, where we use the notation $(\tau)_{\phi}=\phi^{-1} \tau \phi$, ie, if $\tau$ is a Dehn twist along a loop $\delta$ then $(\tau)_{\phi}$ is the Dehn twist along the loop $\phi(\delta)$.

Definition 5 A factorization $F=\tau_{1} \cdot \ldots \cdot \tau_{r}$ in $\mathrm{Map}_{g}$ is an ordered tuple of positive Dehn twists. We say that two factorizations are Hurwitz equivalent $\left(F \sim F^{\prime}\right)$ if they can be obtained from each other by a sequence of Hurwitz moves.

A Lefschetz fibration is thus characterized by a factorization of the identity element in $\mathrm{Map}_{g}$, uniquely determined up to Hurwitz equivalence and simultaneous conjugation of all factors by a same element of $\mathrm{Map}_{g}$, ie, up to the equivalence relation generated by the moves

$$
\tau_{1} \cdot \ldots \cdot \tau_{i} \cdot \tau_{i+1} \cdot \ldots \cdot \tau_{r} \longleftrightarrow \tau_{1} \cdot \ldots \cdot \tau_{i+1} \cdot\left(\tau_{i}\right)_{\tau_{i+1}} \cdot \ldots \cdot \tau_{r} \quad \forall 1 \leq i<r,
$$




$$
\tau_{1} \cdot \ldots \cdot \tau_{r} \longleftrightarrow\left(\tau_{1}\right)_{\phi} \cdot \ldots \cdot\left(\tau_{r}\right)_{\phi} \quad \forall \phi \in \operatorname{Map}_{g}
$$

We will actually be considering Lefschetz fibrations equipped with a distinguished section. The section determines a marked point in each fiber, and so we can lift the monodromy to a relative mapping class group. In fact, even though the normal bundle to the section $s$ is not trivial (it has degree $-k$ for some $k \geq 1$ ), we can restrict ourselves to the preimage of a large disc $\Delta$ containing all the critical values of $f$, and fix a trivialization of the normal bundle to $s$ over $\Delta$. Deleting a small tubular neighborhood of the section $s$, we can now view the monodromy of $f$ as a homomorphism

$$
\psi: \pi_{1}\left(\Delta \backslash\left\{q_{1}, \ldots, q_{r}\right\}\right) \rightarrow \operatorname{Map}_{g, 1},
$$

where $\operatorname{Map}_{g, 1}$ is the mapping class group of a genus $g$ surface with one boundary component. The product of the Dehn twists $\tau_{i}=\psi\left(\gamma_{i}\right)$ is not the identity, but the central element $T_{\delta}^{k} \in \operatorname{Map}_{g, 1}$, where $T_{\delta}$ is the boundary twist, ie, the Dehn twist along a loop parallel to the boundary.

With this understood, a Lefschetz fibration with a distinguished section of square $-k$ is described by a factorization of $T_{\delta}^{k}$ as a product of positive Dehn twists in $\operatorname{Map}_{g, 1}$, up to Hurwitz equivalence and global conjugation.

A word about notations: while we use the multiplicative notation for factorizations, and sometimes write $\tau_{1} \cdot \ldots \cdot \tau_{r}=T_{\delta}^{k}$ to express the fact that $\tau_{1} \cdot \ldots \cdot \tau_{r}$ is a factorization of $T_{\delta}^{k}$, it is important not to confuse a factorization (a tuple of Dehn twists) with the product of its factors (an element in Map $_{g, 1}$ ). We will also use multiplicative notation for the concatenation of factorizations $\left(F \cdot F^{\prime}\right.$ is the factorization consisting of the factors in $F$, followed by those in $F^{\prime}$, and $(F)^{n}$ is the concatenation of $n$ copies of $F$ ), and we will denote by $(F)_{\phi}$ the factorization obtained by conjugating each factor of $F$ by the element $\phi \in \operatorname{Map}_{g, 1}$.

To finish this section, we establish the following properties of Hurwitz equivalence for factorizations of central elements:

Lemma 6 Let $T$ be a central element in a group $G$. Then:

(a) if $F^{\prime} \cdot F^{\prime \prime}$ is a factorization of $T$, then $F^{\prime \prime} \cdot F^{\prime}$ is also a factorization of $T$, and $F^{\prime} \cdot F^{\prime \prime} \sim F^{\prime \prime} \cdot F^{\prime}$;

(b) if $F$ is a factorization of $T$ whose factors generate $G$, then $F \sim(F)_{\phi}$ for all $\phi \in G$;

(c) if $F$ is a factorization of $T$, and $F^{\prime}$ is any factorization, then $F^{\prime} \cdot F \sim F \cdot F^{\prime}$. 
Proof (see also Lemma 6 in [1]).

(a) To prove that any cyclic permutation of the factors amounts to a Hurwitz equivalence, it suffices to prove that if $\tau \in G$ and $\tau \cdot F^{\prime \prime}$ is a factorization of $T$ then $\tau \cdot F^{\prime \prime} \sim F^{\prime \prime} \cdot \tau$. Denote by $\phi$ the product of the factors in $F^{\prime \prime}$ : using Hurwitz moves to move all the factors in $F^{\prime \prime}$ to the left of $\tau$, we have $\tau \cdot F^{\prime \prime} \sim F^{\prime \prime} \cdot(\tau)_{\phi}$. The result then follows from the observation that $\phi=\tau^{-1} T$ commutes with $\tau$.

(b) Let $\tau$ be any of the factors in $F$ : then by (a) we can perform a cyclic permutation of the factors and obtain a factorization $F^{\prime}$ such that $F \sim F^{\prime} \cdot \tau$. Moving $\tau$ to the left of $F^{\prime}$, we have $F^{\prime} \cdot \tau \sim \tau \cdot\left(F^{\prime}\right)_{\tau}=\left(\tau \cdot F^{\prime}\right)_{\tau}$. Applying (a) again we have $\left(\tau \cdot F^{\prime}\right)_{\tau} \sim(F)_{\tau}$. So, for any factor $\tau$ of $F$, we have $F \sim(F)_{\tau}$, and similarly $F \sim(F)_{\tau^{-1}}$. The result then follows from the assumption that the factors of $F$ generate $G$, by expressing $\phi$ in terms of the factors.

(c) Simply move all the factors of $F$ to the left of the factors in $F^{\prime}$, to obtain $F^{\prime} \cdot F \sim F \cdot\left(F^{\prime}\right)_{T}=F \cdot F^{\prime}$ (since $T$ is central).

\section{Universal positive factorizations}

Let us first recall a presentation of $\operatorname{Map}_{g, 1}$ due to Matsumoto [6], which is a reformulation of Wajnryb's classical presentation [10 in a form that is more convenient for our purposes (see Theorem 1.3 and Remark 1.1 of [] ):

Theorem 7 (Matsumoto) For $g \geq 2$, the mapping class group Map $_{g, 1}$ is generated by the Dehn twists $a_{0}, \ldots, a_{2 g}$ along the loops $c_{0}, \ldots, c_{2 g}$ represented in Figure 1, with the relations:

(i) $a_{i} a_{j}=a_{j} a_{i}$ if $c_{i} \cap c_{j}=\emptyset$, and $a_{i} a_{j} a_{i}=a_{j} a_{i} a_{j}$ if $c_{i} \cap c_{j} \neq \emptyset$;

(ii) $\left(a_{0} a_{2} a_{3} a_{4}\right)^{10}=\left(a_{0} a_{1} a_{2} a_{3} a_{4}\right)^{6}$;

(iii) for $g \geq 3:\left(a_{0} a_{1} a_{2} a_{3} a_{4} a_{5} a_{6}\right)^{9}=\left(a_{0} a_{2} a_{3} a_{4} a_{5} a_{6}\right)^{12}$.

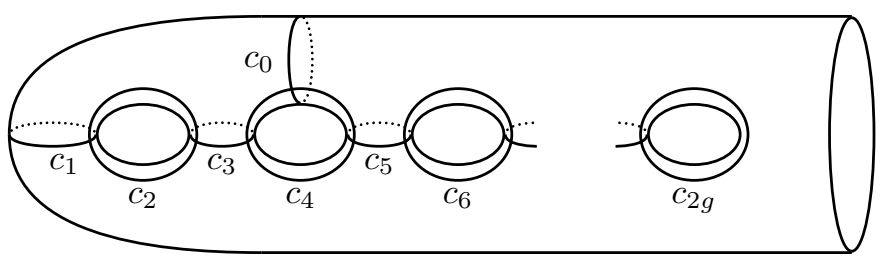

Figure 1: The Dehn-Lickorish-Humphries generators of $\operatorname{Map}_{g, 1}$ 
The relations (i) are the braid relations, and realize $\operatorname{Map}_{g, 1}$ as a quotient of an Artin group, while (ii) is a reformulation of the chain relation, and (iii) is a reformulation of the lantern relation (see [6]).

The subgroup of $\mathrm{Map}_{g, 1}$ generated by $a_{1}, \ldots, a_{2 g}$ is the hyperelliptic subgroup, and is closely related to the braid group $B_{2 g+1}$ (realizing the genus $g$ surface as a double cover of the disc branched in $2 g+1$ points, the Dehn twists $a_{1}, \ldots, a_{2 g}$ are the lifts of the standard generators of $\left.B_{2 g+1}\right)$.

Lemma 8 For every integer $1<n<2 g$, let

$$
\begin{aligned}
R_{n}=\left(a_{n+1} \cdot a_{n+2} \cdot \ldots \cdot a_{2 g}\right)^{2 g-n+1} . & \\
& \cdot \prod_{i=n}^{1}\left(a_{i} \cdot a_{i+1} \cdot \ldots \cdot a_{i+2 g-n}\right) \cdot \prod_{i=2 g-n+1}^{1}\left(a_{i} \cdot a_{i+1} \cdot \ldots \cdot a_{i+n-1}\right) .
\end{aligned}
$$

Then $\left(a_{1} \cdot \ldots \cdot a_{n-1}\right)^{2 n} \cdot\left(R_{n}\right)^{2}$ is a factorization of $T_{\delta}$ in $\operatorname{Map}_{g, 1}$.

Proof We work in the braid group $B_{2 g+1}$ with generators $x_{1}, \ldots, x_{2 g}$, and consider the expression obtained from $R_{n}$ after replacing each $a_{i}$ by $x_{i}$. Then it is easy to see that $a^{\prime}=\left(x_{1} \ldots x_{n-1}\right)^{n}$ is the full twist rotating the $n$ leftmost strands by $2 \pi$, while $a^{\prime \prime}=\left(x_{n+1} \ldots x_{2 g}\right)^{2 g-n+1}$ is the full twist rotating the $2 g+$ $1-n$ rightmost strands by $2 \pi$. Moreover, $b^{\prime}=\prod_{i=n}^{1}\left(x_{i} \ldots x_{i+2 g-n}\right)$ is the braid which exchanges the $n$ leftmost strands with the $2 g-n+1$ rightmost strands in the counterclockwise direction, while $b^{\prime \prime}=\prod_{i=2 g-n+1}^{1}\left(x_{i} \ldots x_{i+n-1}\right)$ does the same for the $2 g-n+1$ leftmost strands and the $n$ rightmost strands. Hence the product $b^{\prime} b^{\prime \prime}$ corresponds to a full rotation of the $n$ leftmost strands around the $2 g-n+1$ rightmost strands, and $a^{\prime} a^{\prime \prime} b^{\prime} b^{\prime \prime}$ is the full twist $\Delta^{2}=\left(x_{1} \ldots x_{2 g}\right)^{2 g+1}$. Since $\Delta^{2}$ is a central element in $B_{2 g+1}$, we also have $a^{\prime \prime} b^{\prime} b^{\prime \prime} a^{\prime}=\Delta^{2}$.

We now lift things to the double cover; since $\Delta^{2}$ lifts to the hyperelliptic element $H$ (rotating the surface about its central axis by $\pi$ ), we deduce from the above calculation that $\left(a_{1} \cdot \ldots \cdot a_{n-1}\right)^{n} \cdot R_{n}$ and $R_{n} \cdot\left(a_{1} \cdot \ldots \cdot a_{n-1}\right)^{n}$ are factorizations of $H$, and hence that $\left(a_{1} \cdot \ldots \cdot a_{n-1}\right)^{n} \cdot\left(R_{n}\right)^{2} \cdot\left(a_{1} \cdot \ldots \cdot a_{n-1}\right)^{n}$ is a factorization of $H^{2}=T_{\delta}$. Since $T_{\delta}$ is central in $\operatorname{Map}_{g, 1}$, the result follows by Lemma 6(a).

It is in fact not hard to check explicitly that the factorization considered in Lemma 8 is Hurwitz equivalent to the standard hyperelliptic factorization $\left(a_{1} \cdot \ldots \cdot a_{2 g}\right)^{4 g+2}$.

From now on we assume that $g \geq 3$. By Theorem 1.4 of $\left[\underline{6},\left(a_{0} a_{2} a_{3} a_{4}\right)^{10}=\right.$ $\left(a_{0} a_{1} a_{2} a_{3} a_{4}\right)^{6}=\left(a_{1} a_{2} a_{3} a_{4}\right)^{10}$ and $\left(a_{0} a_{1} a_{2} a_{3} a_{4} a_{5} a_{6}\right)^{9}=\left(a_{0} a_{2} a_{3} a_{4} a_{5} a_{6}\right)^{12}=$ 
$\left(a_{1} a_{2} a_{3} a_{4} a_{5} a_{6}\right)^{14}$. Hence, we can define new factorizations of $T_{\delta}$ by substitution into the factorization of Lemma 8

Definition 9 Let $\mathcal{A}=\left(a_{0} \cdot a_{2} \cdot a_{3} \cdot a_{4}\right)^{10} \cdot\left(R_{5}\right)^{2}, \mathcal{B}=\left(a_{0} \cdot a_{1} \cdot a_{2} \cdot a_{3} \cdot a_{4}\right)^{6} \cdot\left(R_{5}\right)^{2}$, $\mathcal{C}=\left(a_{0} \cdot a_{1} \cdot a_{2} \cdot a_{3} \cdot a_{4} \cdot a_{5} \cdot a_{6}\right)^{9} \cdot\left(R_{7}\right)^{2}, \mathcal{D}=\left(a_{0} \cdot a_{2} \cdot a_{3} \cdot a_{4} \cdot a_{5} \cdot a_{6}\right)^{12} \cdot\left(R_{7}\right)^{2}$ (where for $g=3$ we take $R_{7}$ to be the empty factorization), and $\mathcal{F}_{0}=\mathcal{A} \cdot \mathcal{B} \cdot \mathcal{C} \cdot \mathcal{D}$.

$\mathcal{A}, \mathcal{B}, \mathcal{C}, \mathcal{D}$ are factorizations of the central element $T_{\delta}$ in which every factor is one of the $\left(a_{i}\right)_{0 \leq i \leq 2 g}$, and every generator appears at least once (except possibly for $\mathcal{D}$, which does not involve $a_{1}$ when $g=3$ ).

We also define $f_{g}^{0}, f_{g}^{A}, f_{g}^{B}, f_{g}^{C}, f_{g}^{D}$ to be the Lefschetz fibrations with monodromy factorizations $\mathcal{F}_{0}, \mathcal{A}, \mathcal{B}, \mathcal{C}, \mathcal{D}$ respectively (so $f_{g}^{A}, f_{g}^{B}, f_{g}^{C}, f_{g}^{D}$ are irreducible and admit sections of square -1 , while $f_{g}^{0}$ is their fiber sum and admits a section of square -4). Let us mention that, as a consequence of Lemma 6(b), when performing a fiber sum with $f_{g}^{0}$ the choice of the identification diffeomorphism between fibers is irrelevant, and all possible ways in which the fiber sum can be carried out are equivalent.

The factorizations $\mathcal{A}, \mathcal{B}, \mathcal{C}, \mathcal{D}$ form a "universal" set of positive factorizations, in the sense that their factors are exactly the generators of $\operatorname{Map}_{g, 1}$ (out of sequence, and with some repetitions), and every relation in the presentation of Theorem [7 can be interpreted either as a Hurwitz equivalence or as a substitution replacing one of these factorizations by another one of them. We will see below that these properties are the key ingredients for the proof of Theorem 2. since many other groups related to braid groups or mapping class groups can be presented in a similar manner, the methods used here may also be relevant to the study of factorizations in these groups.

\section{Stable equivalence of factorizations}

In this section, we prove the following result, which implies Theorem 4

Theorem 10 Let $F, F^{\prime}$ be two factorizations of the same element of $\operatorname{Map}_{g, 1}$ as a product of positive Dehn twists along non-separating curves. Then there exist integers $a, b, c, d, k, l$ such that $F \cdot(\mathcal{A})^{a} \cdot(\mathcal{B})^{b} \cdot(\mathcal{C})^{c} \cdot(\mathcal{D})^{d} \sim F^{\prime} \cdot(\mathcal{A})^{a+l}$. $(\mathcal{B})^{b-l} \cdot(\mathcal{C})^{c+k} \cdot(\mathcal{D})^{d-k}$.

In order to prove this result, we consider factorizations where the factors are either positive Dehn twists or their inverses, and the equivalence relation $\equiv$ generated by the following moves: 
- Hurwitz moves involving only positive Dehn twists;

- creation or cancellation of pairs of inverse factors: $a_{i} \cdot a_{i}^{-1} \equiv a_{i}^{-1} \cdot a_{i} \equiv \emptyset$;

- defining relations of the mapping class group: $a_{i} \cdot a_{j} \equiv a_{j} \cdot a_{i}$ if $c_{i} \cap c_{j}=\emptyset$, $a_{i} \cdot a_{j} \cdot a_{i} \equiv a_{j} \cdot a_{i} \cdot a_{j}$ if $c_{i} \cap c_{j} \neq \emptyset,\left(a_{0} \cdot a_{2} \cdot a_{3} \cdot a_{4}\right)^{10} \equiv\left(a_{0} \cdot a_{1} \cdot a_{2} \cdot a_{3} \cdot a_{4}\right)^{6}$, and $\left(a_{0} \cdot a_{1} \cdot a_{2} \cdot a_{3} \cdot a_{4} \cdot a_{5} \cdot a_{6}\right)^{9} \equiv\left(a_{0} \cdot a_{2} \cdot a_{3} \cdot a_{4} \cdot a_{5} \cdot a_{6}\right)^{12}$.

Lemma 11 If the factors of $F$ are Dehn twists along non-separating curves, then there exists a factorization $\bar{F}$ in which every factor is of the form $a_{i}^{ \pm 1}$ for some $0 \leq i \leq 2 g$, and such that $F \equiv \bar{F}$.

Proof We use pair creations and Hurwitz moves to replace every factor in $F$ by a factorization involving only the $a_{i}^{ \pm 1}$. Let $\tau$ be a factor in $F$. Since $\tau$ is a Dehn twist along a non-separating curve, there exist $g_{0}, \ldots, g_{k} \in\left\{a_{0}^{ \pm 1}, \ldots, a_{2 g}^{ \pm 1}\right\}$ such that $\tau=\left(\prod_{1}^{k} g_{j}\right)^{-1} g_{0}\left(\prod_{1}^{k} g_{j}\right)$. We proceed by induction on $k$. If $k=0$ then $\tau$ is already one of the generators. Otherwise, if $g_{k}$ is one of the generators, say $a_{i}$, then we can write $\tau=\left(a_{i}^{-1} \tilde{\tau} a_{i}\right) \equiv a_{i}^{-1} \cdot a_{i} \cdot\left(a_{i}^{-1} \tilde{\tau} a_{i}\right) \equiv a_{i}^{-1} \cdot \tilde{\tau} \cdot a_{i}$ (using a pair creation and a Hurwitz move). Similarly, if $g_{k}=a_{i}^{-1}$, then we can write $\tau=\left(a_{i} \tilde{\tau} a_{i}^{-1}\right) \equiv\left(a_{i} \tilde{\tau} a_{i}^{-1}\right) \cdot a_{i} \cdot a_{i}^{-1} \equiv a_{i} \cdot \tilde{\tau} \cdot a_{i}^{-1}$. Since $\tilde{\tau}$ is conjugated to one of the generators by a word of length $k-1$, this completes the proof.

Lemma 12 Under the assumptions of Theorem $10, F \equiv F^{\prime}$.

Proof We first use Lemma 11 to replace $F$ and $F^{\prime}$ by equivalent factorizations $\bar{F}$ and $\bar{F}^{\prime}$ whose factors are all of the form $a_{i}^{ \pm 1}$. Next, recall that if a group $G$ admits a presentation with generators $\left\{a_{i}, i \in I\right\}$ and relations $\left\{r_{j}, j \in J\right\}$, then it is generated as a monoid by the elements $\left\{a_{i}, a_{i}^{-1}, i \in I\right\}$, and a presentation of $G$ as a monoid is given by the set of relations $R^{\prime}=\left\{r_{j}, j \in J\right\} \cup$ $\left\{a_{i} a_{i}^{-1}=1, a_{i}^{-1} a_{i}=1, i \in I\right\}$. Hence, if $\bar{F}$ and $\bar{F}^{\prime}$ are factorizations of the same element, then we can rewrite one into the other by successively applying the rewriting rules given by the set of relations $R^{\prime}$. However, in the case of the mapping class group, each rewriting is one of the moves that generate the equivalence relation $\equiv$ (either one of the defining relations of $\operatorname{Map}_{g, 1}$, or the creation or cancellation of a pair of inverses).

Denote by $\equiv^{+}$the equivalence relation generated by Hurwitz moves and by the defining relations, ie, without allowing creations of pairs of inverse factors. Then we have: 
Lemma 13 Under the assumptions of Theorem 10, there exists an integer $n$ such that $F \cdot(\mathcal{A})^{n} \equiv^{+} F^{\prime} \cdot(\mathcal{A})^{n}$.

Proof By Lemma 12 $F \equiv F^{\prime}$, so we can transform $F$ into $F^{\prime}$ by a sequence of Hurwitz moves, pair creations/cancellations, and defining relations. Call $F=$ $F_{0}, F_{1}, \ldots, F_{m}=F^{\prime}$ the successive factorizations appearing in this sequence of moves; let $n_{j}$ be the number of factors of the form $a_{i}^{-1}$ appearing in the factorization $F_{j}$, and let $n=\sup \left\{n_{0}, \ldots, n_{m}\right\}$.

Recall that the factors of $\mathcal{A}$ generate $\mathrm{Map}_{g, 1}$; therefore, by Lemma 6(a), for every $i \in\{0, \ldots, 2 g\}$ there exists a factorization $\mathcal{A}_{i}$ whose factors are elements of $\left\{a_{0}, \ldots, a_{2 g}\right\}$, and such that $\mathcal{A} \sim a_{i} \cdot \mathcal{A}_{i} \sim \mathcal{A}_{i} \cdot a_{i}$. (For example $\mathcal{A}_{i}$ can be obtained by cyclically permuting the factors of $\mathcal{A}$ and deleting an occurrence of $\left.a_{i}\right)$. Let $F_{j}^{+}$be the factorization obtained from $F_{j}$ by replacing each factor of the form $a_{i}^{-1}$ by the factorization $\mathcal{A}_{i}$. Then we claim that, for all $0 \leq j<m$, $F_{j}^{+} \cdot(\mathcal{A})^{n-n_{j}} \equiv^{+} F_{j+1}^{+} \cdot(\mathcal{A})^{n-n_{j+1}}$.

Indeed, if $F_{j+1}$ is obtained from $F_{j}$ by a Hurwitz move or by applying a defining relation, then the negative factors are not involved and the claim is obvious. If $F_{j+1}$ is obtained from $F_{j}$ by deleting a pair of mutually inverse factors $a_{i} \cdot a_{i}^{-1}$, $F_{j+1}^{+}$is obtained from $F_{j}^{+}$by deleting an occurrence of the subword $a_{i} \cdot \mathcal{A}_{i}$. Hence, we can write $F_{j}^{+}=F_{j}^{\prime} \cdot a_{i} \cdot \mathcal{A}_{i} \cdot F_{j}^{\prime \prime}$ and $F_{j+1}^{+}=F_{j}^{\prime} \cdot F_{j}^{\prime \prime}$ for some $F_{j}^{\prime}, F_{j}^{\prime \prime}$, and the claim follows from the sequence of Hurwitz moves

$$
F_{j}^{\prime} \cdot a_{i} \cdot \mathcal{A}_{i} \cdot F_{j}^{\prime \prime} \cdot(\mathcal{A})^{n-n_{j}} \sim F_{j}^{\prime} \cdot \mathcal{A} \cdot F_{j}^{\prime \prime} \cdot(\mathcal{A})^{n-n_{j}} \sim F_{j}^{\prime} \cdot F_{j}^{\prime \prime} \cdot(\mathcal{A})^{n-n_{j}+1},
$$

where in the last step we have used Lemma 6(c). The argument is the same for creations of pairs of inverses. The proof is then completed by observing that $F_{0}^{+}=F$ and $F_{m}^{+}=F^{\prime}$, since $F$ and $F^{\prime}$ contain no negative factors.

We can now proceed with the proof of Theorem 10. By Lemma 13, there exists $n$ such that $F \cdot(\mathcal{A})^{n} \equiv^{+} F^{\prime} \cdot(\mathcal{A})^{n}$, so we can transform $F \cdot(\mathcal{A})^{n}$ into $F^{\prime} \cdot(\mathcal{A})^{n}$ by a sequence of Hurwitz moves and applications of the defining relations. Let $F_{0}=F \cdot(\mathcal{A})^{n}, F_{1}, \ldots, F_{m}=F^{\prime} \cdot(\mathcal{A})^{n}$ be the successive factorizations appearing in this sequence of moves. If $F_{j+1}$ is obtained from $F_{j}$ by a Hurwitz move, or by applying one of the braid relations, then we have $F_{j+1} \sim F_{j}$. For example, a braid relation of the form $a_{i} \cdot a_{j} \cdot a_{i} \equiv a_{j} \cdot a_{i} \cdot a_{j}$ can be viewed as a succession of two Hurwitz moves $a_{i} \cdot a_{j} \cdot a_{i} \sim a_{j} \cdot\left(a_{i}\right) a_{j} \cdot a_{i} \sim a_{j} \cdot a_{i} \cdot\left(a_{i}\right) a_{j} a_{i}$, where $\left(a_{i}\right)_{a_{j} a_{i}}=\left(a_{j} a_{i}\right)^{-1} a_{i} a_{j} a_{i}=a_{j}$.

On the other hand, if $F_{j+1}$ is obtained from $F_{j}$ by applying the relation (ii) from Theorem [7, then we can write $F_{j}=F_{j}^{\prime} \cdot\left(a_{0} \cdot a_{2} \cdot a_{3} \cdot a_{4}\right)^{10} \cdot F_{j}^{\prime \prime}$ for some 
$F_{j}^{\prime}, F_{j}^{\prime \prime}$, and $F_{j+1}=F_{j}^{\prime} \cdot\left(a_{0} \cdot a_{1} \cdot a_{2} \cdot a_{3} \cdot a_{4}\right)^{6} \cdot F_{j}^{\prime \prime}$. It is then easy to check, using Lemma 6 (a) and (c), that $F_{j} \cdot \mathcal{B} \sim F_{j+1} \cdot \mathcal{A}$; and vice-versa if we apply relation (ii) backwards. Similarly, if $F_{j+1}$ is obtained from $F_{j}$ by applying relation (iii), then $F_{j} \cdot \mathcal{D} \sim F_{j+1} \cdot \mathcal{C}$, and vice-versa if we apply relation (iii) backwards.

Hence, if we concatenate each $F_{j}$ with suitable numbers of copies of $\mathcal{A}, \mathcal{B}, \mathcal{C}$ and $\mathcal{D}$ (depending on $j$ ), then we can realize each step as a Hurwitz equivalence. Since we always trade a copy of $\mathcal{A}$ for a copy of $\mathcal{B}$, and a copy of $\mathcal{C}$ for a copy of $\mathcal{D}$, Theorem 10 follows.

We can now prove Theorem 4

Proof of Theorem 4 Let $F$ be a factorization in $\mathrm{Map}_{g, 1}$ associated to the Lefschetz fibration $f$ : then the product of the factors in $F$ is equal to $T_{\delta}^{m}$, for some integer $m \geq 1$ (such that the chosen section of $f$ has self-intersection $-m)$. The result then follows by applying Theorem 10 to $F$ and $F^{\prime}=(\mathcal{A})^{m}$.

\section{Proof of Theorem 2}

Let $F$ and $F^{\prime}$ be factorizations in $\operatorname{Map}_{g, 1}$ describing the monodromies of the Lefschetz fibrations $f$ and $f^{\prime}$. Assumption (ii) on the self-intersection numbers of the distinguished sections implies that the products of the factors in $F$ and $F^{\prime}$ are equal to each other, and are of the form $T_{\delta}^{m}$ for some $m \geq 1$. We first deal with the reducible singular fibers, using the following lemma:

Lemma 14 If $\tau, \tau^{\prime}$ are Dehn twists along separating curves of the same type, then there exists an integer $n$ and a factorization $F^{\prime \prime}$ involving only Dehn twists along non-separating curves, such that $\tau \cdot(\mathcal{A})^{n} \sim \tau^{\prime} \cdot F^{\prime \prime}$.

Proof $\tau, \tau^{\prime}$ are conjugated to each other in $\operatorname{Map}_{g, 1}$, so there exist $g_{1}, \ldots, g_{k} \in$ $\left\{a_{0}^{ \pm 1}, \ldots, a_{2 g}^{ \pm 1}\right\}$ such that $\tau^{\prime}=\left(\prod_{1}^{k} g_{j}\right)^{-1} \tau\left(\prod_{1}^{k} g_{j}\right)$. It is enough to consider the case $k=1$ (iterating $k$ times in the general case). If $\tau^{\prime}=a_{i}^{-1} \tau a_{i}$ then, with the same notations as in the proof of Lemma 13, we have $\tau \cdot \mathcal{A} \sim \tau \cdot a_{i} \cdot \mathcal{A}_{i} \sim a_{i} \cdot \tau^{\prime} \cdot \mathcal{A}_{i} \sim$ $\tau^{\prime} \cdot\left(a_{i}\right)_{\tau^{\prime}} \cdot \mathcal{A}_{i}$, and the result follows by setting $F^{\prime \prime}=\left(a_{i}\right)_{\tau^{\prime}} \cdot \mathcal{A}_{i}$. Similarly, if $\tau^{\prime}=a_{i} \tau a_{i}^{-1}$ then $\tau \cdot \mathcal{A} \sim \mathcal{A} \cdot \tau \sim \mathcal{A}_{i} \cdot a_{i} \cdot \tau \sim \mathcal{A}_{i} \cdot \tau^{\prime} \cdot a_{i} \sim \tau^{\prime} \cdot\left(\mathcal{A}_{i}\right)_{\tau^{\prime}} \cdot a_{i}$.

The manner in which we use this lemma is the following: let $s$ be the number of reducible singular fibers of $f$ and $f^{\prime}$. Without loss of generality, we can assume that the homologically trivial vanishing cycles correspond to the first 
$s$ factors of $F$ and $F^{\prime}$, and that they are ordered according to types (this can always be ensured by performing Hurwitz moves). Call these factors $\tau_{1}, \ldots, \tau_{s}$ for $F$, and $\tau_{1}^{\prime}, \ldots, \tau_{s}^{\prime}$ for $F^{\prime}$. Then assumption (iii) on the numbers of reducible singular fibers implies that $\tau_{j}$ and $\tau_{j}^{\prime}$ are conjugated for each $1 \leq j \leq s$. Hence, applying Lemma 14 to each pair $\left(\tau_{j}, \tau_{j}^{\prime}\right)$, and adding sufficiently many copies of $\mathcal{A}$ to $F$ (using Lemma 6) (c) to move them to the beginning of the factorization), we can replace each $\tau_{j}$ by $\tau_{j}^{\prime}$, at the expense of generating extra Dehn twists along nonseparating curves. After suitable Hurwitz moves, we conclude that there exists an integer $N$ and factorizations $\tilde{F}, \tilde{F}^{\prime}$ involving only Dehn twists along non-separating curves, such that $F \cdot(\mathcal{A})^{N} \sim \tau_{1}^{\prime} \cdot \ldots \cdot \tau_{s}^{\prime} \cdot \tilde{F}$ and $F^{\prime} \cdot(\mathcal{A})^{N} \sim \tau_{1}^{\prime} \cdot \ldots \cdot \tau_{s}^{\prime} \cdot \tilde{F}^{\prime}$.

Since $\tilde{F}$ and $\tilde{F}^{\prime}$ are factorizations of the same element $\left(\tau_{1}^{\prime} \ldots \tau_{s}^{\prime}\right)^{-1} T_{\delta}^{m+N}$, we can apply Theorem 10 to them. It follows that there exist integers $a, b, c, d, k, l$ such that $F \cdot(\mathcal{A})^{N+a} \cdot(\mathcal{B})^{b} \cdot(\mathcal{C})^{c} \cdot(\mathcal{D})^{d} \sim F^{\prime} \cdot(\mathcal{A})^{N+a+l} \cdot(\mathcal{B})^{b-l} \cdot(\mathcal{C})^{c+k} \cdot(\mathcal{D})^{d-k}$. This implies that the fiber sums $\hat{f}=f \#(N+a) f_{g}^{A} \# b f_{g}^{B} \# c f_{g}^{C} \# d f_{g}^{D}$ and $\hat{f}^{\prime}=f^{\prime} \#(N+a+l) f_{g}^{A} \#(b-l) f_{g}^{B} \#(c+k) f_{g}^{C} \#(d-k) f_{g}^{D}$ are isomorphic. Performing additional fiber sums if necessary, we can assume that $N+a=b=$ $c=d$. Then, in order to complete the proof of Theorem 2 it is sufficient to prove that $k=l=0$. For this purpose we use the following lemmas to compare the Euler-Poincaré characteristics and signatures of the total spaces $\hat{M}$ and $\hat{M}^{\prime}$ of $\hat{f}$ and $\hat{f}^{\prime}$ :

Lemma $15 \chi\left(\hat{M}^{\prime}\right)-\chi(\hat{M})=\chi\left(M^{\prime}\right)-\chi(M)+10 l-9 k$.

Proof Recall that the Euler characteristic of a genus $g$ Lefschetz fibration over $S^{2}$ with $r$ singular fibers is equal to $4-4 g+r$. Hence, we just have to compare the numbers of singular fibers of $\hat{f}$ and $\hat{f}^{\prime}$. Since $f_{g}^{A}$ has 10 more singular fibers than $f_{g}^{B}$, and $f_{g}^{C}$ has 9 fewer singular fibers than $f_{g}^{D}$, the result follows.

Lemma $16 \sigma\left(\hat{M}^{\prime}\right)-\sigma(\hat{M})=\sigma\left(M^{\prime}\right)-\sigma(M)-6 l+5 k$.

Proof By Novikov additivity, it is sufficient to show that the signatures of the total spaces $M_{A}, M_{B}, M_{C}, M_{D}$ of $f_{g}^{A}, f_{g}^{B}, f_{g}^{C}, f_{g}^{D}$ satisfy the relations $\sigma\left(M_{A}\right)=$ $\sigma\left(M_{B}\right)-6$ and $\sigma\left(M_{C}\right)=\sigma\left(M_{D}\right)+5$.

These signatures can be computed explicitly via an algorithm due to Ozbagci 8 . Since Ozbagci's formula is a sum of individual contributions which each depend only on one of the factors and on the product of all the preceding factors, it 
is sufficient to carry out the algorithm for the portions of $\mathcal{A}$ and $\mathcal{B}$ (resp. $\mathcal{C}$ and $\mathcal{D}$ ) which differ from each other; the contributions from the common part $\left(R_{5}\right)^{2}$ (resp. $\left.\left(R_{7}\right)^{2}\right)$ will be the same in both cases.

In fact, after a closer look at the signature formula it is easy to convince oneself that $\sigma\left(M_{A}\right)-\sigma\left(M_{B}\right)$ and $\sigma\left(M_{C}\right)-\sigma\left(M_{D}\right)$ do not depend on $g$, and can be computed for a fixed low value of $g$ (e.g., $g=3$ ).

Then, rather than Ozbagci's somewhat complicated formula, one can use the following simple recipe to determine the signature - the underlying principle being that, given a Lefschetz fibration $f: M \rightarrow S^{2}$ admitting a section, the complement to the fiber and section classes in $H_{2}(M, \mathbb{Z})$ is generated by certain linear combinations of the Lefschetz thimbles of $f$.

Given the set of vanishing cycles $\left(\delta_{1}, \ldots, \delta_{r}\right)$ (ie, loops in the fiber $\Sigma_{g}$ such that each monodromy factor $\tau_{i}$ is the Dehn twist along $\delta_{i}$ ), form the $r \times r$ matrix $Q$ whose entries are given by

$$
q_{i j}= \begin{cases}0 & \text { if } i>j \\ -1 & \text { if } i=j \\ \delta_{i} \cdot \delta_{j} & \text { if } i>j\end{cases}
$$

where $\delta_{i} \cdot \delta_{j}$ is the intersection number in $H_{1}\left(\Sigma_{g}, \mathbb{Z}\right)$. In a suitable sense, $Q$ is the matrix of the intersection pairing on the space of formal linear combinations of Lefschetz thimbles, and its antisymmetrization $A=Q-Q^{t}$ describes the intersection pairing between vanishing cycles inside $\Sigma_{g}$.

Viewing $Q$ and $A$ as bilinear forms, the kernel of $A$ is the space of all combinations of Lefschetz thimbles which have homologically trivial boundary in $H_{1}\left(\Sigma_{g}, \mathbb{Z}\right)$, and can hence be completed to 2 -cycles inside $M$. The restriction $Q^{\prime}=Q_{\mid \operatorname{Ker} A}$ is now a (degenerate) symmetric bilinear form, of rank $b_{2}(M)-2$; and $Q^{\prime}$ has the same signature as the intersection form on $H_{2}(M, \mathbb{Z})$, ie $\sigma\left(Q^{\prime}\right)=\sigma(M)$.

Applying this formula, we easily check that for $g=3, \sigma\left(M_{A}\right)=-48, \sigma\left(M_{B}\right)=$ $-42, \sigma\left(M_{C}\right)=-35$, and $\sigma\left(M_{D}\right)=-40$.

The proof of Theorem 2 can now be completed by observing that, since $\chi\left(M^{\prime}\right)=$ $\chi(M)$ and $\sigma\left(M^{\prime}\right)=\sigma(M)$ by assumption (i), and since $\hat{M}$ and $\hat{M}^{\prime}$ are diffeomorphic by construction, we must have $10 l=9 k$ and $6 l=5 k$, which implies that $k=l=0$. 


\section{References}

[1] D Auroux, Fiber sums of genus 2 Lefschetz fibrations, Turkish J. Math. 27 (2003) 1-10, arXiv:math.GT/0204285 MathReview

[2] S K Donaldson, Lefschetz pencils on symplectic manifolds, J. Differential Geom. 53 (1999) 205-236 MathReview

[3] H Endo, Meyer's signature cocycle and hyperelliptic fibrations, Math. Ann. 316 (2000) 237-257 MathReview

[4] A Kas, On the handlebody decomposition associated to a Lefschetz fibration, Pacific J. Math. 89 (1980) 89-104 MathReview

[5] V Kharlamov, V Kulikov, On braid monodromy factorizations, Izv. Ross. Akad. Nauk Ser. Mat. 67 (2003) 79-118; translation: Izvestia Math. 67 (2003) 499-534, arXiv:math.AG/0302113 MathReview

[6] M Matsumoto, A presentation of mapping class groups in terms of Artin groups and geometric monodromy of singularities, Math. Ann. 316 (2000) 401418 MathReview

[7] B Moishezon, Complex surfaces and connected sums of complex projective planes, Lecture Notes in Math. 603, Springer-Verlag (1977) MathReview

[8] B Ozbagci, Signatures of Lefschetz fibrations, Pacific J. Math. 202 (2002) 99118 MathReview

[9] B Siebert, G Tian, On the holomorphicity of genus two Lefschetz fibrations, Ann. Math. to appear, arXiv:math.SG/0305343

[10] B Wajnryb, A simple presentation for the mapping class group of an orientable surface, Israel J. Math. 45 (1983) 157-174; errata: J S Birman, B Wajnryb, Israel J. Math. 88 (1994) 425-427 MathReview 